\title{
Expression of SOFAT by T- and B-lineage cells may contribute to bone loss
}

\author{
CHRISTIAN R. JARRY ${ }^{1}$, ELIZABETH F. MARTINEZ ${ }^{2}$, DAIANE C. PERUZZO ${ }^{1}$, VANESSA CARREGARO ${ }^{3}$, \\ LAÍS A. SACRAMENTO ${ }^{3}$, VERA C. ARAÚJO ${ }^{2}$, M. NEALE WEITZMANN ${ }^{4,5}$ and MARCELO H. NAPIMOGA ${ }^{6}$ \\ ${ }^{1}$ Periodontal Medicine Research Group; ${ }^{2}$ Laboratory of Oral Pathology, São Leopoldo Mandic Institute and Research Center, \\ Campinas, São Paulo 13045-755; ${ }^{3}$ Department of Biochemistry and Immunology, Ribeirão Preto Medical School, \\ University of São Paulo, São Paulo 14049-900, Brazil; ${ }^{4}$ Atlanta Department of Veterans Affairs Medical Center, Decatur, \\ GA 30033; ${ }^{5}$ Department of Medicine, Division of Endocrinology, Metabolism and Lipids, Emory University, \\ Atlanta, GA 30322, USA; ${ }^{6}$ Laboratory of Immunology and Molecular Biology, \\ São Leopoldo Mandic Institute and Research Center, Campinas, \\ São Paulo 13045-755, Brazil
}

Received December 9, 2015; Accepted March 2, 2016

DOI: $10.3892 / \mathrm{mmr} .2016 .5045$

\begin{abstract}
A novel T cell-secreted cytokine, termed secreted osteoclastogenic factor of activated $\mathrm{T}$ cells (SOFAT) that induces osteoclastic bone resorption in a RANKL-independent manner, has been described. Our group have previously reported that SOFAT is highly expressed in gingival tissues of patients with chronic periodontitis suggesting a putative role in the bone loss associated with periodontal disease. The aim of the present study was to identify other potential cellular sources of SOFAT in the bone resorptive lesions of patients with periodontal disease. Gingival tissues were biopsied from systemically healthy subjects without periodontal disease $(n=5)$ and patients with chronic periodontitis $(n=5)$, and the presence of SOFAT was analyzed by immunohistochemistry and immunofluorescence staining. The present data demonstrated marked SOFAT staining in diseased periodontal tissues that was predominantly associated with the lymphocytic infiltration of gingival tissues. Notably, in addition to $\mathrm{CD}^{+}$ $\mathrm{T}$ cells, B-lineage cells including plasma cells also exhibited strong staining for SOFAT. As SOFAT has not previously been reported in B-lineage cells, splenic T cells and B cells were further purified from BALB/c mice and activated using CD3/CD28 and lipopolysaccharide, respectively. SOFAT was quantified by reverse transcription-quantitative polymerase chain reaction and was shown to be significantly expressed
\end{abstract}

Correspondence to: Dr Marcelo H. Napimoga, Laboratory of Immunology and Molecular Biology, São Leopoldo Mandic Institute and Research Center, 13 José Rocha Junqueira, Campinas, São Paulo 13045-755, Brazil

E-mail: marcelo.napimoga@gmail.com; napimogamh@yahoo.com

Key words: secreted osteoclastogenic factor of activated $\mathrm{T}$ cells, osteoclast, inflammation, periodontitis, B cell, T cell
$(\mathrm{P}<0.05)$ in both activated $\mathrm{T}$ cells and $\mathrm{B}$ cells compared with unstimulated cells. These data support a putative role of SOFAT in the bone loss associated with chronic periodontal disease. In addition, to the best of our knowledge, this study demonstrates for the first time that in addition to $\mathrm{T}$ cells, B-lineage cells may also be a significant source of SOFAT in inflammatory states.

\section{Introduction}

Periodontitis is an inflammatory lesion that is accompanied by soft tissue destruction and bone resorption in the tooth-supporting structures. There is a balance between bone resorption by osteoclasts and bone formation by osteoblasts, which determines the level of bone mass. A shift in this balance increasing the number of osteoclasts results in alveolar bone destruction $(1,2)$. Although bacterial infection underlies periodontal disease, recent studies have begun to emphasize the fundamental role of inflammation in the pathologic process $(3,4)$. This is exemplified by studies in which suppression of the host response using inhibitors of inflammation or genetic manipulation of inflammatory pathways in animal models, attenuate periodontal bone loss. Notably, in the absence of an aggressive host response, the severity of bone loss stimulated by periodontal pathogens is typically reduced (5).

In this context, a deeply rooted interaction between the immune system and the skeleton (the immuno-skeletal interface) is now well described, a field referred to as 'osteoimmunology'. The identification of the receptor activator of NF- $\kappa$ B (RANK)/RANK ligand (RANKL)/osteoprotegerin (OPG) system has been crucial in the understanding of osteoclast biology (6). When RANKL, the key osteoclastogenic cytokine binds to its receptor RANK on osteoclast precursors, they differentiate into bone resorbing osteoclasts. This process is moderated by OPG, a RANKL decoy receptor. These concepts are supported by genetic studies in mice in 
which knockout mice deficient in RANKL or RANK, not only present with osteopetrotic phenotypes, but also lack peripheral lymph nodes (7). This supports the hypothesis that RANKL and RANK are essential in osteoclastogenesis and immune organogenesis (8). Although numerous inflammatory cytokines promote osteoclastogenesis, these factors all converge on the RANKL/RANK/OPG system, which, at least under physiological conditions, is considered to be the final downstream effector of osteoclastogenesis and bone resorption (9).

An active periodontal lesion is characterized by the prominent infiltration of B cells and T cells $(10,11)$. Notably, bacterial antigen presentation by professional antigen presenting cells (APCs), such as dendritic cells, B cells and macrophages, induce effector $\mathrm{T}$ lymphocytes to express membrane bound and soluble forms of RANKL. Bacterial antigen-engagement of the B cell receptor (BCR) induces B cells to produce not only the IgG antibody but also RANKL. Furthermore, the activated antigen-specific $\mathrm{T}$ cells provide $\mathrm{B}$ cell co-stimulatory signals through CD40/CD40L ligation, which also is considered to upregulate RANKL expression from B cells (12), as well as promote the production of OPG $(13,14)$.

Consequently, RANKL is considered to be of fundamental importance to bone biology under physiological conditions and in pathological inflammatory states. However, it has been reported that activated $\mathrm{T}$ cells also produce another less-well characterized factor, secreted osteoclastogenic factor of activated T cells (SOFAT), a RANKL-independent osteoclastogenic cytokine that may contribute to bone loss in inflammatory conditions including rheumatoid arthritis $(15,16)$ and chronic periodontitis (17).

Although SOFAT was first identified in the conditioned medium of activated $\mathrm{T}$ cells and demonstrated by us to be highly upregulated in diseased periodontal tissues (15), the potential for other sources of SOFAT has not been investigated. In the present study immunohistochemical staining was performed to demonstrate SOFAT expression in diseased periodontal tissues and reverse transcription-quantitative polymerase chain reaction (RT-qPCR) was conducted to quantify SOFAT expression in activated murine T cells and B cells. To the best of our knowledge, this study reports for the first time that human and mouse cells of the B-lineage are also a significant source of SOFAT when activated in vitro, and that human B cells and plasma cells express SOFAT in the context of periodontal infection.

\section{Materials and methods}

Subjects. Five systemically healthy subjects without periodontitis and 5 systemically healthy individuals with generalized chronic periodontitis were selected from the patients of the Periodontal Clinic of São Leopoldo Mandic (São Paulo, Brazil). Subjects who fulfilled the inclusion/exclusion criteria described below were invited to enter the study. All eligible subjects were informed of the nature, potential risks and benefits of their participation in the study and signed informed consent. This study was approved by the São Leopoldo Mandic Institutional Review Board (IRB-\#811.702).

Inclusion and exclusion criteria. Subjects aged 35-60 years and presenting with at least 15 teeth (excluding third molars) were eligible for this study. Periodontitis patients had generalized chronic periodontitis (18), with $>30 \%$ of the sites presenting concomitant probing depth (PD) and clinical attachment level $(\mathrm{CAL}) \geq 4 \mathrm{~mm}$ and, at least one tooth indicated for extraction due to advanced periodontitis [sites with PD and CAL $>7 \mathrm{~mm}$ with bleeding on probing (BoP) and mobility]. Patients without-periodontitis subjects had no sites with CAL $>3 \mathrm{~mm}$ and $<30 \%$ of sites presenting BoP and/or marginal bleeding (MB). Subjects were selected from patients undergoing periodontal esthetic surgery (gingivoplasty) for the correction of gingival discrepancies and asymmetries.

Patients with any systemic condition that could affect the progression of periodontal disease (e.g. immunological disorders, diabetes mellitus, bone-related diseases and complications, such as osteoporosis, ankyosing spondylitis, recent bone fractures and rheumatoid arthritis) and long-term administration of anti-inflammatory agents (e.g. glucocorticoids), use of immunosuppressive medications, hormone replacement therapy and antiresorptive agents (e.g. bisphosphonates and denosumab), lactation, current smoking and smoking within the preceding 5 years, periodontal and/or antibiotic therapies in the previous 6 months and use of mouth rinses containing antimicrobials in the preceding 2 months were excluded from the study. Subjects presenting use of orthodontic appliances or periapical pathologies related to endodontic lesions were also excluded.

Clinical examinations. One examiner (Dr Peruzzo), using a previously described calibration method (19), performed all clinical examinations. This trained examiner was able to provide reproducible measurements of under $0.5 \mathrm{~mm}$. The clinical parameters, registered dichotomously (i.e. BoP), were calculated by the Kappa-Light test (19), using GraphPad Prism 5.0 (GraphPad Software, Inc., La Jolla, CA, USA) and the intra-examiner agreement was $>0.92$. The following parameters were assessed at six sites of all teeth (mesio-buccal, medio-buccal, disto-buccal, mesio-lingual, medio-lingual, disto-lingual), excluding third molars using a manual periodontal probe (UNC15; Hu-Friedy, Chicago, IL, USA): Visible plaque accumulation (PI), marginal bleeding (MB), BoP, PD (mm) and CAL (mm) (20).

Gingival biopsies. Gingival samples were collected 1 week after clinical examination so as not to modify the nature of the periodontal inflammatory process. For the periodontitis group, the gingival tissues were collected during tooth extraction due to advanced periodontitis ( $\mathrm{PD}$ and $\mathrm{CAL} \geq 7 \mathrm{~mm}$ with $\mathrm{BoP}$ ) to obtain representative areas of inflamed periodontal tissue. If the patient had two or more teeth with these characteristics, a gingival biopsy from only one tooth with the worst clinical diagnosis was included. For the non-periodontitis group, gingival biopsies were collected from a single tooth without signs of clinical inflammation ( $\mathrm{PD} \leq 3 \mathrm{~mm}$ without $\mathrm{BoP}$ and/or $\mathrm{MB})$, indicated for gingivoplasty due to aesthetic reasons. All gingival samples included junctional and sulcular epitheliums and adjacent connective gingival tissue.

Immunohistochemistry. The samples were rinsed with saline solution and immediately fixed in $10 \%$ buffered neutral formalin for $48 \mathrm{~h}$. The biopsies were embedded in paraffin 
Table I. Demographics and full-mouth clinical data of healthy subjects and patients with periodontitis.

\begin{tabular}{lccc}
\hline Characteristic & Healthy & Diseased & P-value \\
\hline $\mathrm{N}$ & 5 & 5 & $\mathrm{NS}$ \\
Age (years, mean $\pm \mathrm{SD}$ ) & $30.4 \pm 3.6$ & $35.7 \pm 2.1$ & $\mathrm{NS}$ \\
Gender (male/female, n) & $3 / 2$ & $2 / 3$ & $\mathrm{NS}$ \\
Race-Caucasian & 5 & 5 & $\mathrm{NS}$ \\
Number of teeth present (mean \pm SD) & $27.10 \pm 1.70$ & $25.34 \pm 3.17$ & $<0.0001$ \\
Plaque index $(\%$ mean \pm SD) & $10.21 \pm 3.21$ & $68.97 \pm 17.56$ & $<0.0001$ \\
Marginal bleeding (\% mean \pm SD) & $6.45 \pm 5.22$ & $41.38 \pm 19.54$ & $<.25 \pm 2.90$
\end{tabular}

SD, standard deviation; NS, non-significant.

to obtain longitudinal step-serial sections $(3 \mu \mathrm{m})$. Every fifth section was stained with hematoxylin and eosin (H\&E; Dinâmica, São Paulo, Brazil) to observe the presence of all histological structures (e.g., epithelium and connective tissue) using a Bioval ${ }^{\circledR}$ L-2000A microscope (Bioval, Jiangbei, China). Two slices of the tissue obtained from the section before and two slices obtained from the section after the H\&E sections were selected for immunohistochemical analysis, to certify that the slices used were close to those that were observed in the H\&E and immunohistochemistry. The histological sections were mounted on glass slides pretreated with 3-aminopropyltriethoxy-silane, deparaffinized and hydrated. Antigen retrieval was achieved after treatment with citric acid ( $\mathrm{pH}$ 6.0) for $30 \mathrm{~min}$ at $95^{\circ} \mathrm{C}$. Subsequently, sections were treated with $20 \%$ hydrogen peroxide (Dinâmica) for $30 \mathrm{~min}$ to eliminate endogenous peroxidase and then washed with Tris $0.05 \mathrm{M}(\mathrm{pH}$ 7.4). The sections were incubated with polyclonal rabbit antiSOFAT primary antibody (1:100; Rheabiotech, Campinas, Brazil) for $1 \mathrm{~h}$ at room temperature. Sections were then washed with tap water and incubated with enzyme-conjugated polymer backbone containing secondary antibody molecules (Envision Dual Link; Dako, Carpinteria, CA, USA) for $30 \mathrm{~min}$ at room temperature in a humidified chamber. After another washing step (with tap water), the sections were incubated with 3,3'-diaminobenzidine tetrahydrochloride (DAB; Dako) for $10 \mathrm{~min}$ at room temperature in a humidified chamber and counter-stained with Mayer's hematoxylin (Dinâmica). The sections were mounted in Permount (Thermo Fisher Scientific, Inc., Waltham, MA, USA). Digital photomicrography was obtained with a Zeiss Axioskop 2 plus microscope equipped with AxioCam digital camera using Axiovision application software (Carl Zeiss, Gottingen, Germany).

Indirect immunofluorescence. Sections $(3 \mu \mathrm{m})$ were fixed in methanol for $6 \mathrm{~min}$ at $-20^{\circ} \mathrm{C}$, rinsed in phosphate-buffered saline (PBS) and then blocked using $1 \%$ bovine serum albumin (Sigma-Aldrich, St. Louis, MO, USA) in PBS at room temperature for $30 \mathrm{~min}$. The primary antibodies used were as follows: Polyclonal rabbit anti-SOFAT primary antibody $(1: 100$; Rheabiotech, Campinas, Brazil), and monoclonal mouse
anti-CD3 (1:100; cat. no. M7254; Dako, Glostrup, Denmark), monoclonal mouse anti-CD45RO (1:100; cat. no. M0742; Dako), monoclonal mouse anti-CD20 (1:100; cat. no. M0755; Dako) and anti-plasma cell (1:100; cat. no. M7077; Dako). The control staining reaction was performed using PBS as a substitute for the primary antibody. The secondary antibody used was either Alexa Fluor 594 goat anti-mouse IgG antibody or Alexa Fluor 488 goat anti-rabbit IgG antibody (both Molecular Probes, Eugene, OR, USA) for $30 \mathrm{~min}$ at room temperature. After washing, the preparations were mounted using Vectashield with 4/-6-diamidino-2-phenylindole (both from Vector Laboratories Ltd., Peterborough, UK) and observed under a conventional Zeiss Axioskop 2 fluorescence microscope (Carl Zeiss) equipped with a 63x Plan Apochromatic 1.4NA and 100x Plan Apochromatic 1.4NA lenses under standard conditions. Immunofluorescence was repeated three times for each antibody.

Cell culture. BALB/c mice (weight, 20-25 g) were housed in temperature-controlled rooms $\left(22-25^{\circ} \mathrm{C}\right)$ with access to water and food ad libitum. All experiments were conducted in accordance with the National Institutes of Health guidelines for the welfare of experimental animals and with the approval of the Ethics Committee of the Faculty of Medicine of Ribeirão Preto (University of São Paulo, São Paulo, Brazil). The mice were only used in a single experimental group and were terminally anesthetized by inhalation of $3 \%$ isoflurane (Cristália, São Paulo, Brazil) followed by cervical dislocation. Single-cell suspensions of spleens from naïve $\mathrm{BALB} / \mathrm{c}$ mice were prepared aseptically, red blood cells were lysed and $\mathrm{T}$ and $\mathrm{B}$ cells were isolated using MACS beads Multisort (Miltenyi Biotec, Teterow, Germany) according to the manufacturer's instructions. Cells were dispensed into 48-well plates in a total volume of $500 \mu$ l complete RPMI-1640 medium (1x10 cells/well; Gibco; Thermo Fisher Scientific, Inc.) and stimulated with plate-bound anti-CD3 monoclonal antibody $(5 \mu \mathrm{g} / \mathrm{ml})$ and anti-CD28 $(5 \mu \mathrm{g} / \mathrm{ml})$ for $\mathrm{T}$ cell stimulation and $200 \mathrm{ng} / \mathrm{ml}$ lipopolysaccharide (LPS; Sigma-Aldrich) for B cell stimulation or medium as negative control. mRNA was harvested after $24 \mathrm{~h}$ of culture at 
A

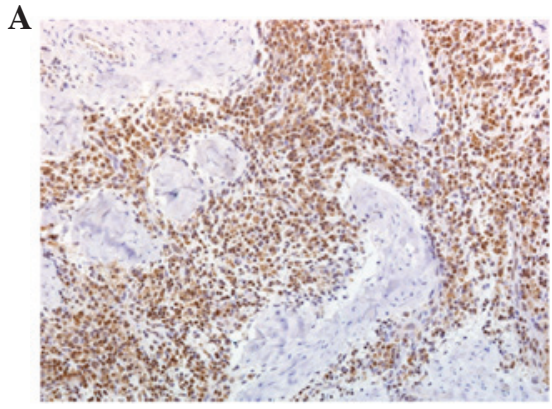

C

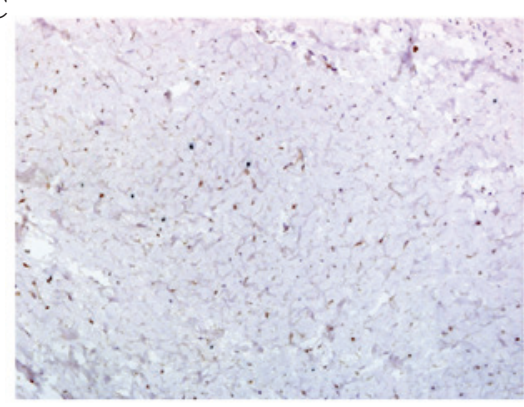

B

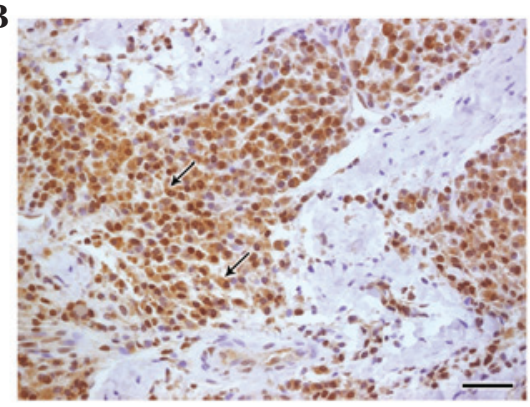

D

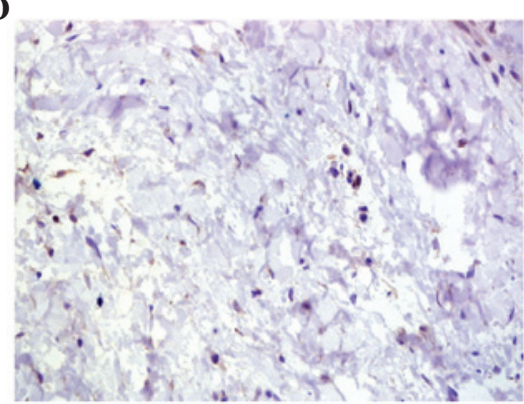

Figure 1. Immunohistochemical analyses of SOFAT expression in gingival tissue samples. Gingival tissue samples from healthy patients stained with rabbit polyclonal IgG anti-SOFAT at (A) magnification, x200 and (B) magnification x400. The arrows indicate plasma cells. (C) Gingival tissue samples from patients with chronic periodontitis stained with rabbit polyclonal IgG anti-SOFAT, observed at (C) magnification, $\mathrm{x} 200$ and (D) magnification, $\mathrm{x} 400$. SOFAT, secreted osteoclastogenic factor of activated T cells.

$37^{\circ} \mathrm{C}$ in $5 \% \mathrm{CO}_{2}$, using RNeasy Mini kit (Qiagen, Valencia, CA, USA). The RNA was quantified with a NanoVue spectrophotometer (GE Healthcare, Bjorkgatan, Sweden). Aliquots of $1 \mu \mathrm{g}$ total RNA from each sample were treated with DNase I (Thermo Fisher Scientific, Inc.) and used for reverse transcription reactions with the Superscript III First Strand cDNA Synthesis kit (Thermo Fisher Scientific, Inc.), according to the manufacturer's instructions. qPCR was performed using a 7500 Fast Real Time PCR system (Thermo Fisher Scientific, Inc.) with SYBR Green (Thermo Fisher Scientific, Inc.) as detection dye. The primers (Invitrogen; Thermo Fisher Scientific, Inc.) were as follows: Forward, 5'-ATGGACATTATCGTTCTGCTGCCC-3' and reverse, 5'-CTGGGAGGTGTTGAGGGCATG-3' for SOFAT; and forward, 5'-TGGCCTCCAAGGAGTAAGAAAC-3' and reverse 5'-TGGAAATTGTGAGGGAGATGCT-3' for GAPDH, which served as the internal gene reference. The cycling conditions were $10 \mathrm{~min}$ at $95^{\circ} \mathrm{C}$ followed by 40 cycles of $95^{\circ} \mathrm{C}$ for $15 \mathrm{sec}$ and $60^{\circ} \mathrm{C}$ for $1 \mathrm{~min}$. The quantification data were analyzed with the SDS System Software version 2.0.6 (Thermo Fisher Scientific, Inc.) and the relative expression levels were calculated according to the Comparative $\mathrm{Cq}$ method, as $2^{-\Delta \Delta \mathrm{Cq}}(21)$.

Statistical analysis. Data were examined for normality using the Shapiro-Wilk test. Results were expressed as the mean \pm standard deviation. Independent t-test was used for differences in the age and number of teeth, whereas the variable gender and race was analyzed using Fisher's exact test. Mann-Whitney test was used to perform intragroup comparisons regarding $\mathrm{PI}, \mathrm{MB}, \mathrm{BoP}, \mathrm{PD}$ and CAL. To analyze the results, one-way analysis of variance with post hoc Bonferroni test was applied. All statistical analyses were performed with the JMP Software statistical software (SAS Institute, Cary,
NC, USA). $\mathrm{P}<0.05$ was considered to indicate a statistically significant difference.

\section{Results}

Patient characteristics. The demographics and clinical status of the subjects are shown in Table I. No significant difference was observed between healthy and diseased subjects regarding age, gender, race and number of teeth present. Data analysis demonstrated significantly increased levels of PI, MB, BoP, $\mathrm{PD}$ and $\mathrm{CAL}$ in diseased subjects compared with healthy subjects $(\mathrm{P}<0.001)$.

Immunohistochemical analysis of SOFAT expression. Using immunohistochemistry to analyze SOFAT expression in healthy and diseased periodontal tissues, it was revealed that marked lymphoid infiltration was present in patients with periodontal disease with almost all of these lymphocytes expressed SOFAT protein (Fig. 1 panels A and B). By contrast, in healthy gingival tissues, small number of lymphocytes were observed, although few of these cells expressed SOFAT (Fig. 1 panels $\mathrm{C}$ and D). Based on morphological characteristics it was demonstrated that plasma cells also stained positive for SOFAT expression (Fig. 1, panel B, arrows).

Expression of SOFAT in different cells types. To conclusively verify that cell types other than $\mathrm{T}$ cells express SOFAT in inflamed gingival tissue, double-color immunofluorescence (Fig. 2) was used to identify SOFAT (green) and lymphocyte-phenotypic makers (red). Images of SOFAT and CD marker-positive cells were merged and double-positive cells were observed as yellow/orange staining. The data revealed significant SOFAT expression in these cellular infiltrates, particularly by memory $\mathrm{T}$ cells $(\mathrm{CD} 45 \mathrm{RO})$ and by $\mathrm{B}$ cells 


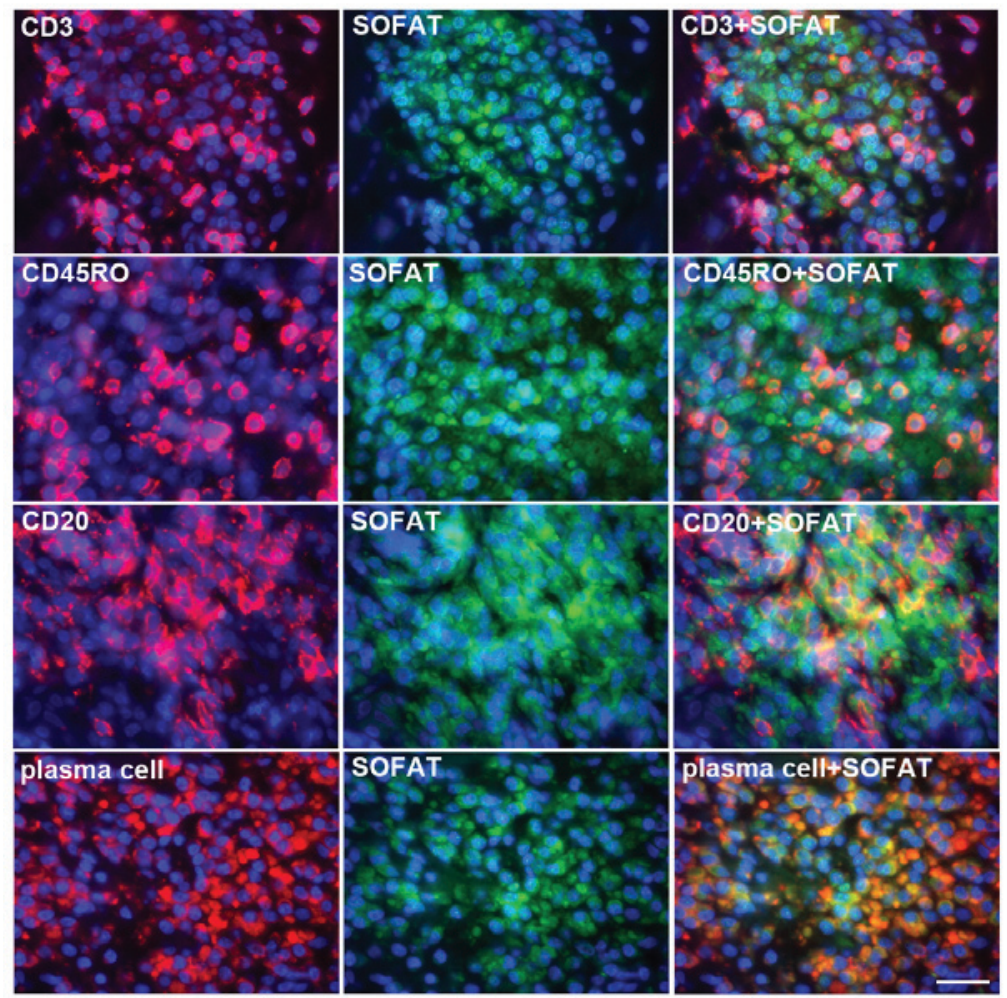

Figure 2. Indirect immunofluorescence for SOFAT (green) and for CD3, CD45RO, CD20 or plasma cells (red) in gingival tissue from patients with chronic periodontitis. Specific CD marker-stained cells and SOFAT-stained cells were merged and visualized as orange (merged; right column). Scale bar, $100 \mu \mathrm{m}$. SOFAT, secreted osteoclastogenic factor of activated T cells.

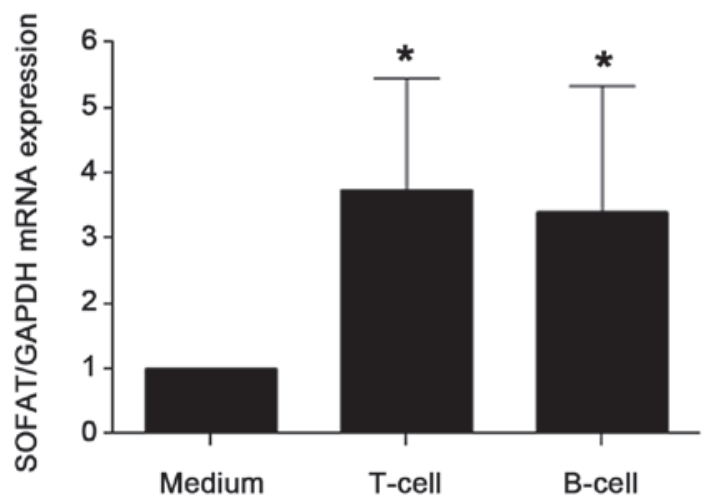

Figure 3. SOFAT mRNA expression from purified splenic T cells and B cells from $\mathrm{BALB} / \mathrm{c}$ mice activated using lipopolysaccharide or $\mathrm{CD} 3 / \mathrm{CD} 28$. ${ }^{*} \mathrm{P}<0.05$, compared with control, as determined by one-way analysis of variance followed by Bonferroni post hoc test. SOFAT, secreted osteoclastogenic factor of activated $\mathrm{T}$ cells.

(CD20). In addition there was significant colocalization of plasma cell markers. Although the majority of B-lineage cells were positive for SOFAT staining, not all $\mathrm{T}$ cells expressed SOFAT.

SOFAT $m R N A$ expression in B cells and $T$ cells. To further demonstrate that beside T cells, activated B cells are also an important source of SOFAT, lymphocytes from mouse spleen were activated in vitro using CD3/CD28 for T cells and LPS a potent activator of B cells. As shown in Fig. 3, in comparison to non-stimulated splenocytes, activated $\mathrm{T}$ cells and $\mathrm{B}$ cells showed a significant increase in the expression of SOFAT mRNA $(\mathrm{P}<0.05)$. No significant difference in the magnitude of SOFAT mRNA expression between T cells and B cells was identified.

\section{Discussion}

The cells of the immune system are widely distributed throughout the body. When an infection occurs, the inflammatory response allows marshaling of immune system elements to specific sites. Typically, early events in the inflammatory reaction to infection are not detectable clinically. As the infectious process becomes more chronic, clinically evident inflammation occurs, generating high levels of cytokines and other mediators of inflammation associated with activation of the periodontal response. Cytokines are important in the modulation of inflammatory and pro-resorptive cells. Several molecules have been described in the literature to be involved in periodontal disease progression $(5,9,22,23)$. One such factor is RANKL, a cytokine that promotes the differentiation of osteoclast precursor cells and is critical in periodontal bone resorption (9). However, although RANKL is considered to be a key cytokine for physiological osteoclastogenesis and is important in periodontal bone erosion, other cytokines may amplify periodontal bone loss that is driven by RANKL. In fact, the identification of novel RANKL-independent activities in culture medium conditioned by activated $\mathrm{T}$ cells has been reported in a previous series of studies (15). This molecule that also potently induces osteoblastic IL-6 production (24) was later identified and termed SOFAT (16). 
It was demonstrated that patients with chronic periodontitis express high levels of SOFAT in diseased periodontal tissues, and that injection of recombinant RANKL into periodontal tissue leads to significant erosion of alveolar bone in vivo (17). Additionally, it was recently demonstrated that the mechanism of action of SOFAT is RANKL-independent, indicating that, by co-opting osteoblasts to increase osteoclastogenic cytokine production, SOFAT may exacerbate inflammation, and support osteoclast formation and bone destruction (25). These data suggest that SOFAT may significantly contribute to alveolar bone loss in periodontal infection and in other inflammatory states.

The results of the present investigation extend current understanding of SOFAT biology and to the best of our knowledge is the first study to show that not only $\mathrm{T}$ cells, but also mature B cells, and terminally differentiated plasma cells are key sources of SOFAT in conditions of periodontal infection. These findings in periodontal tissues isolated from individuals with periodontitis suggested that activated lymphocytes in general appear to be a significant source of SOFAT in chronic periodontitis and may contribute to bone resorption in periodontal disease.

There is a marked presence of B lymphocytes and plasma cells in the gingival tissue of patients with periodontal diseases and antigen-specific T-cell and B-cell activation in the swollen gingival tissue is required for the induction of periodontal bone loss. In fact, simple inflammation induced by LPS alone does not cause periodontal bone resorption suggesting that a robust host response may underlie bone damage. Indeed an animal model involving adoptive transfer of Aggregatibacter actinomycetemcomitans-responsive B cells exhibited significantly increased levels of bone resorption compared with non-responsive B cells. Indeed, A. actinomycetemcomitans-binding and RANKL-expressing B cells were recovered from the gingival tissues of recipient rats transferred A. actinomycetemcomitans-responsive B cells (26). These data suggest that B lymphocytes can contribute to increased periodontal bone resorption during immune responses to A. actinomycetemcomitans in periodontal disease and this effect is associated with the upregulation of RANKL expression. Indeed, T cells and B cells have been reported to aid in periodontal bone loss though RANKL production (27).

Data shown in the present study revealed that B cells, in addition to $\mathrm{T}$ cells, are important cellular sources of bone destructive factors SOFAT and RANKL in human periodontal disease lesions. In conclusion, the prominent expression of SOFAT by $\mathrm{B}$ and $\mathrm{T}$ cells in the chronic periodontitis lesion likely augments RANKL-induced bone resorption, and the use of RANKL-targeting pharmacological anti-resorptive agents alone, may be insufficient to prevent bone loss in periodontal infection owing to the action of SOFAT.

\section{Acknowledgements}

The authors would like to thank Pollyanna Tombini Montaldi, Nadir S. Freitas and Jeruza P. Bossonaro for their technical expertise and assistance. This study was supported by grants from the FAPESP (grant no. 2013/09524-2); CNPq (grant no. 470780/2013-4) and CNPq (Researcher Scholarship to MHN; grant no. 303555/2013-0).

\section{References}

1. Udagawa N, Takahashi N, Jimi E, Matsuzaki K, Tsurukai T, Itoh K, Nakagawa N, Yasuda H, Goto M, Tsuda E, et al: Osteoblasts/stromal cells stimulate osteoclast activation through expression of osteoclast differentiation factor/RANKL but not macrophage colony-stimulating factor: Receptor activator of NF-kappa B ligand. Bone 25: 517-523, 1999.

2. Suda T, Takahashi N, Udagawa N, Jimi E, Gillespie MT and Martin TJ: Modulation of osteoclast differentiation and function by the new members of the tumor necrosis factor receptor and ligand families. Endocr Rev 20: 345-357, 1999.

3. Graves DT, Li J and Cochran DL: Inflammation and uncoupling as mechanisms of periodontal bone loss. J Dent Res 90: 143-153, 2011.

4. Khalaf H, Lönn J and Bengtsson T: Cytokines and chemokines are differentially expressed in patients with periodontitis: Possible role for TGF- $\beta 1$ as a marker for disease progression. Cytokine 67: 29-35, 2014.

5. Graves DT, Fine D, Teng YT, Van Dyke TE and Hajishengallis G: The use of rodent models to investigate host-bacteria interactions related to periodontal diseases. J Clin Periodontol 35: 89-105, 2008.

6. Lacey DL, Timms E, Tan HL, Kelley MJ, Dunstan CR, Burgess T, Elliott R, Colombero A, Elliott G, Scully S, et al: Osteoprotegerin ligand is a cytokine that regulates osteoclast differentiation and activation. Cell 93: 165-176, 1998.

7. Kong YY, Yoshida H, Sarosi I, Tan HL, Timms E, Capparelli C, Morony S, Oliveira-dos-Santos AJ, Van G, Itie A, et al: OPGL is a key regulator of osteoclastogenesis, lymphocyte development and lymph-node organogenesis. Nature 28: 315-323, 1999.

8. Teng YT, Nguyen H, Gao X, Kong YY, Gorczynski RM, Singh B, Ellen RP and Penninger JM: Functional human T-cell immunity and osteoprotegerin ligand control alveolar bone destruction in periodontal infection. J Clin Invest 106: R59-R67, 2000.

9. Chen B, Wu W, Sun W, Zhang Q, Yan F and Xiao Y: RANKL expression in periodontal disease: Where does RANKL come from? Biomed Res Int 2014: 731039, 2014.

10. Seymour GJ and Greenspan JS: The phenotypic characterization of lymphocyte subpopulations in established human periodontal disease. J Periodontal Res 14: 39-46, 1979.

11. Taubman MA, Stoufi ED, Ebersole JL and Smith DJ: Phenotypic studies of cells from periodontal disease tissues. J Periodontal Res 19: 587-590, 1984.

12. Kajiya M, Giro G, Taubman MA, Han X, Mayer MP and Kawai T: Role of periodontal pathogenic bacteria in RANKL-mediated bone destruction in periodontal disease. J Oral Microbiol 8: 2 , 2010.

13. Yun TJ, Chaudhary PM, Shu GL, Frazer JK, Ewings MK, Schwartz SM, Pascual V, Hood LE and Clark EA: OPG/FDCR-1, a TNF receptor family member, is expressed in lymphoid cells and is up-regulated by ligating CD40. J Immunol 161: 6113-6121, 1998.

14. Li Y, Toraldo G, Li A, Yang X, Zhang H, Qian WP and Weitzmann MN: B cells and T cells are critical for the preservation of bone homeostasis and attainment of peak bone mass in vivo. Blood 109: 3839-3848, 2007.

15. Weitzmann MN, Cenci S, Rifas L, Haug J, Dipersio J and Pacifici R: T cell activation induces human osteoclast formation via receptor activator of nuclear factor kappaB ligand-dependent and -independent mechanisms. J Bone Miner Res 16: 328-337, 2001.

16. Rifas L and Weitzmann MN: A novel T cell cytokine, secreted osteoclastogenic factor of activated $\mathrm{T}$ cells, induces osteoclast formation in a RANKL-independent manner. Arthritis Rheum 60: 3324-3335, 2009.

17. Jarry CR, Duarte PM, Freitas FF, de Macedo CG, Clemente-Napimoga JT, Saba-Chujfi E, Passador-Santos F, de Araújo VC and Napimoga MH: Secreted osteoclastogenic factor of activated T cells (SOFAT), a novel osteoclast activator, in chronic periodontitis. Hum Immunol 74: 861-866, 2013.

18. Armitage GC: Development of a classification system for periodontal diseases and conditions. Ann Periodontol 4: 1-6, 1999.

19. Araujo MW, Hovey KM, Benedek JR, Grossi SG, Dorn J, Wactawski-Wende J, Genco RJ and Trevisan M: Reproducibility of probing depth measurement using a constant-force electronic probe: Analysis of inter-and intraexaminer variability. J Periodontol 74: 1736-1740, 2003.

20. Ainamo J and Bay I: Problems and proposals for recording gingivitis and plaque. Int Dent J 25: 229-235, 1975. 
21. Livak KJ and Schmittgen TD: Analysis of relative gene expression data using real-time quantitative PCR and the 2(-Delta Delta C(T)) Method. Methods 25: 402-408, 2001.

22. Chen XT, Tan JY, Lei LH and Chen LL: Cytokine levels in plasma and gingival crevicular fluid in chronic periodontitis. Am J Dent 28: 9-12, 2015.

23. Garlet GP: Destructive and protective roles of cytokines in periodontitis: A re-appraisal from host defense and tissue destruction viewpoints. J Dent Res 89: 1349-1363, 2010.

24. Rifas L and Avioli LV: A novel T cell cytokine stimulates interleukin-6 in human osteoblastic cells. J Bone Miner Res 14 1096-1103, 1999.
25. Napimoga MH, Demasi AP, Jarry CR, Ortega MC, de Araújo VC and Martinez EF: In vitro evaluation of the biological effect of SOFAT on osteoblasts. Int Immunopharmacol 26: 378-383, 2015.

26. Han X, Kawai T, Eastcott JW and Taubman MA: Bacterial-responsive B lymphocytes induce periodontal bone resorption. J Immunol 176: 625-631, 2006.

27. Kawai T, Matsuyama T, Hosokawa Y, Makihira S, Seki M, Karimbux NY, Goncalves RB, Valverde P, Dibart S, Li YP, et al: $\mathrm{B}$ and $\mathrm{T}$ lymphocytes are the primary sources of RANKL in the bone resorptive lesion of periodontal disease. Am J Pathol 169: 987-998, 2006. 